\title{
A Modified Goldfrank Sniffing Bar Improves Accuracy in Identifying Simulated Toxins in a Case-Based Teaching Model
}

\author{
Gerald F. O'Malley, DO, Kenneth R. Deitch, DO, Paul Dominici, MD, Michael Young, DO
}

Department of Emergency Medicine, Albert Einstein Medical Center, Philadelphia, PA

\begin{abstract}
Objective: To determine if olfactory analysis of toxin proxies aid in the identification of poisons when compared to case histories alone. Methods: A convenience sample of 42 emergency medicine residents participated in a prospective single-blind observational study using a sniffing bar designed with the technique described by Goldfrank et.al. Each subject received 12 written cases describing a patient with a chemical exposure. Ten chemicals with odors corresponding to a specific case history and 2 chemical case controls with no odor comprised the sniffing bar. Each subject was asked to identify the toxin first by reading the case history alone, then again after smelling the corresponding odor. All subjects were asked to record their level of confidence for each case before and after the addition of the sniffing bar using a 5-point Likert scale. Percentages correct before and after the sniffing bar intervention were calculated and two-tailed p-values and 95\% confidence intervals were measured. Continuous comparative variables such as the means of the Likert scale confidence measurements were analyzed with the two-tailed $t$-test. Results: Median percentage of toxins correctly identified was 64.3\% (range 9.5-97.6) when based on case description alone and 86.9\% (range 9.5-95.2) with addition of the sniffing bar. The percentage of initially incorrect identification reversed after additional data provided by the sniffing bar was $11.5 \%$ (range 0-14). Of the 10 actual toxins, correct identification of 6 achieved statistical significance after introduction of the sniffing bar ( $\mathrm{p}=0.0017$; 95\% CI 4.6-11.4). There was no association between level of training and improved accuracy with use of the sniffing bar, and overall confidence did not improve significantly after addition of the sniffing bar. Conclusion: Diagnostic accuracy of poisons in case-based scenarios is improved with the use of a sniffing bar. The sniffing bar is a useful adjunct to a traditional case-based emergency medicine toxicology teaching curriculum.
\end{abstract}

\section{INTRODUCTION}

Certain poisonings are associated with specific odors. In 1980, Goldfrank et al. described a "sniffing bar," which is a series of test tubes filled with chemicals that simulate the odors of specific poisons and may be used as a teaching adjunct for toxic odor recognition [1]. ED physicians may be trained to recognize certain odors that might aid in the diagnosis of particular characteristic, corresponding poisons [2]. This study evaluates the usefulness of a modified Goldfrank sniffing bar as an adjunct to the traditional teaching of toxicology to emergency medicine residents.

\section{METHODS}

This was a single blind, observational study at an urban, level one trauma center. A convenience sample of 48 emergency medicine residents in a four-year, ACGME-approved training program participated.

Keywords: odor recognition, poisoning, toxicology, teaching

Notes: There was no outside funding of any kind used for this study.

Corresponding Author: Dr. Gerald Francis O'Malley, DO, Department of Emergency Medicine,Albert Einstein Medical Center 5501 Old York Road, Philadelphia, PA 19141 
All subjects were placed simultaneously in a large, well-ventilated auditorium. Each subject was given 12 written test cases to read. The history in each case described a patient poisoned by a particular chemical with a characteristic odor. The histories and case details were developed using information found in leading toxicology textbooks $[3,4]$. Each case history gave a typical presentation of each type of poisoning. Two identical sniffing bars were placed at opposite ends of the auditorium to avoid crosscontamination by odors wafting in the air. The subjects were asked to read the case histories, and for each case choose the correct diagnosis from a list of five choices (standard multiple choice format). These choices were based only on the information and description in the case history. After choosing their answers, the subjects were asked to record how confident they felt with their diagnosis on a 5 -point Likert scale $(0=$ wild guess, $1=$ not very confident, 2 = somewhat confident, 3 = confident, $4=$ very confident). The subjects were then allowed to smell the contents of the tube corresponding to the case that they were considering. At that point, the subjects had the opportunity to reconsider their initial diagnosis, and change it. The subjects were asked to record how confident they felt with their diagnosis after the additional sniffing bar data was introduced using the same 5-point Likert scale.

The sniffing bar was made from 12 identical test tubes in a test tube holder. We used standard red-top Vacutainer blood collection tubes. The tubes were covered with opaque tape so that the contents were not visible. Each tube was filled with an equal amount of material. Ten of the tubes had chemicals with specific odors. Two of the tubes were chemical case controls, with no odor. Each tube had a rubber stopper, and was labeled 1-12 to correspond with each case. The contents of the tubes, the representative odors, and the simulated poison or chemical are described in Table 1.
The subjects recorded their answers and confidence scores on preprinted data collection sheets. After completing the test, all study materials and answer sheets were turned into the research staff. The subjects were asked not to discuss the test with each other during the examination. No reference materials were allowed during the examination. The subjects were not specifically educated on the poisons represented on the test or on the specific odors associated with these poisons.

\section{DATA ANALYSIS}

Percentages correct before and after the sniffing bar intervention were calculated algebraically, and two-tailed $p$ values and 95\% confidence intervals were measured. Continuous comparative variables such as the means of the Likert scale confidence measurements were analyzed with the two-tailed $t$-test. This was an observational, descriptive study and no sample size calculation was performed.

\section{RESULTS}

All residents participating in this test were training in emergency medicine at a four-year, ACGME-approved training program. Of 48 possible residents, 42 were available on the day of the experiment and served as a convenience sample. Class distribution of the 42 participating residents was similar $(\mathrm{PGY} 1=28.5 \%$, PGY2 $=21.43 \%$, PGY3=21.43\%, PGY4=26.9\%). Percentages of correct toxin identification from the case scenarios pre- and post-sniffing bar test are described in Table 2.

Median percentage of toxins correctly identified improved from $64.3 \%$ (range $9.5-97.6$ ) to $86.9 \%$ (range 9.5-95.2) with addition of the sniffing bar.

\begin{tabular}{llll}
\hline Table 1: & Poisons Comprising the "Sniffing Bar" \\
\hline Tube & Contents of Tube & Representative Odor & Poison/Chemical \\
\hline 1 & Almond extract & Bitter almonds & Cyanide \\
\hline 2 & Macerated peanuts & Peanuts & Vacor \\
\hline 3 & $\begin{array}{l}\text { Household bleach } \\
\text { (sodium hypochlorite) }\end{array}$ & Bleach & Chloramine gas \\
\hline 4 & Sugar water & No odor & Control \\
\hline 5 & N-acetylcysteine & Rotten eggs & Hydrogen sulfide \\
\hline 6 & Vinegar & Vinegar & Clacial acetic acid \\
\hline 7 & Macerated garlic & Garlic oil & Organophospates \\
\hline 8 & $\begin{array}{l}\text { Macerated wintergreen } \\
\text { Life Savers candy }\end{array}$ & Oil of wintergreen & Methyl salicyclate \\
\hline 9 & Sugar water & No odor & Control tube \\
\hline 10 & Household ammonia & Ammonia & Ammonia \\
\hline 11 & Camphor moth balls & Camphor & Mothballs \\
\hline 12 & Paint thinner (toluene) & Paint thinner & Aromatic hydrocarbons \\
\hline & & &
\end{tabular}




\begin{tabular}{|c|c|c|c|c|c|c|c|c|c|c|c|c|}
\hline Test case & 1 & 2 & 3 & 4 & 5 & 6 & 7 & 8 & 9 & 10 & 11 & 12 \\
\hline Pre-odor correct/42 & 29 & 4 & 11 & 11 & 25 & 28 & 41 & 35 & 11 & 23 & 26 & 32 \\
\hline Post-odor correct/42 & 36 & 4 & 7 & 10 & 32 & 37 & 40 & 40 & 10 & 37 & 27 & 38 \\
\hline
\end{tabular}

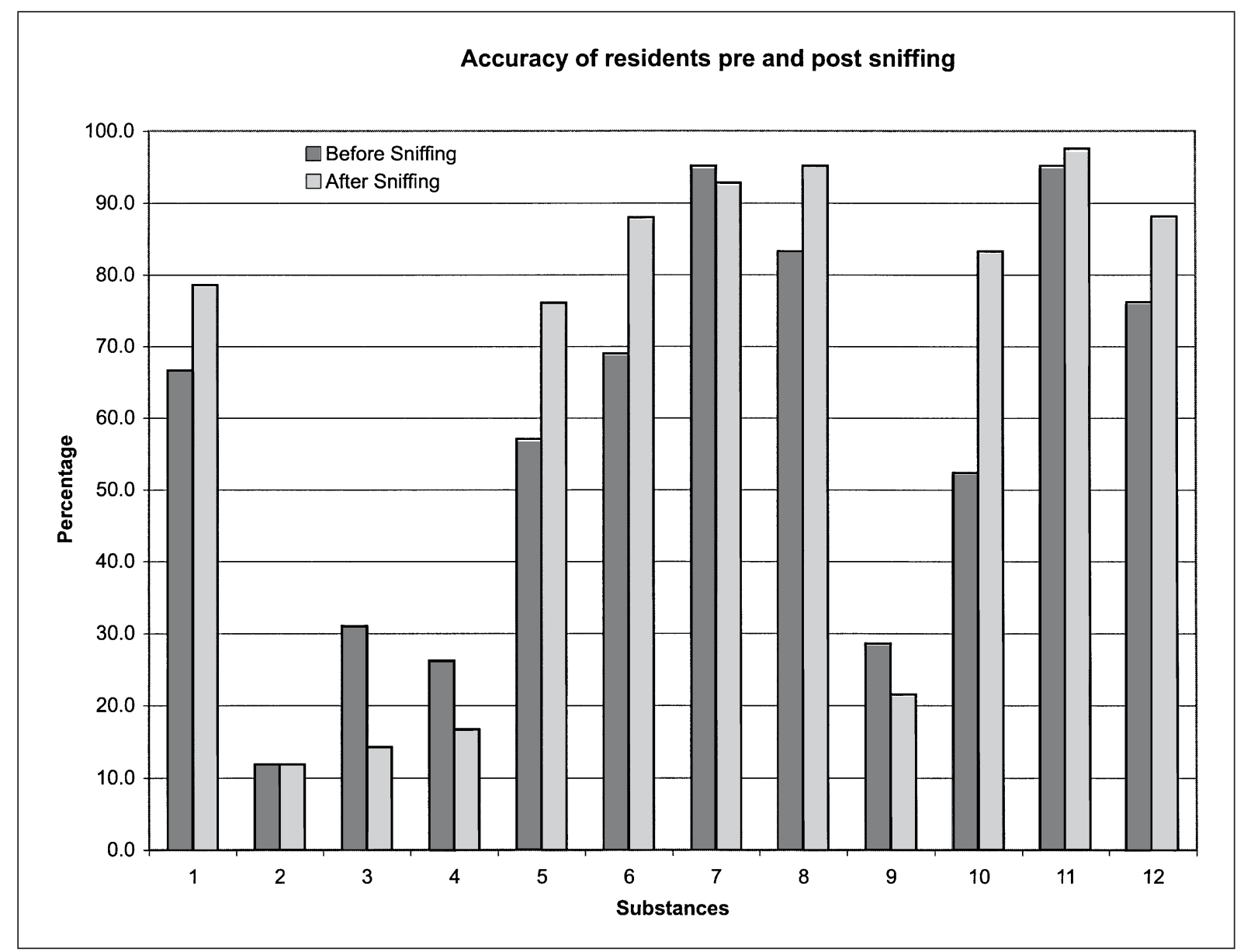

Figure 1: Accuracy of residents pre- and post-sniffing

In the two control cases (numbers 4 and 9) involving the use of sugar water and a generic case history, as expected, there was no significant difference in correct answers pre- and post-sniffing bar. In case number 4, 10 subjects guessed correctly pre-sniffing bar and 11 guessed correctly after the sniffing bar. In case number 9, 11 subjects guessed correctly pre-sniffing bar and 10 guessed correctly after the sniffing bar.

In the ten actual cases, the results can be described in three ways. In six cases (numbers 1, 5, 6, 8, 10, and 12), there was a statistically significant improvement in accuracy after introduction of the sniffing bar $(p=0.0017 ; 95 \% \mathrm{CI}=4.6-11.4)$. In three cases (numbers 2,7 , and 11 ), there was no statistically significant improvement or decline in accuracy after introduction of the sniffing bar ( $p>0.9 ; 95 \% \mathrm{CI}=-2.4-2.8)$.

In one case (number 3 ), there was a decline in accuracy after introduction of the sniffing bar (11 subjects guessed the correct toxin based on the case history, but 4 subjects changed their answers to an incorrect guess after smelling the contents of the corresponding tube in the sniffing bar).

An analysis of the six statistically significant correct responses demonstrated no association between level of training and correct odor identification. There was no significant improvement in overall reported confidence in the diagnosis after addition of the sniffing bar (average difference in Likert score $=0.49$ [CI 0.79-0.19]. 


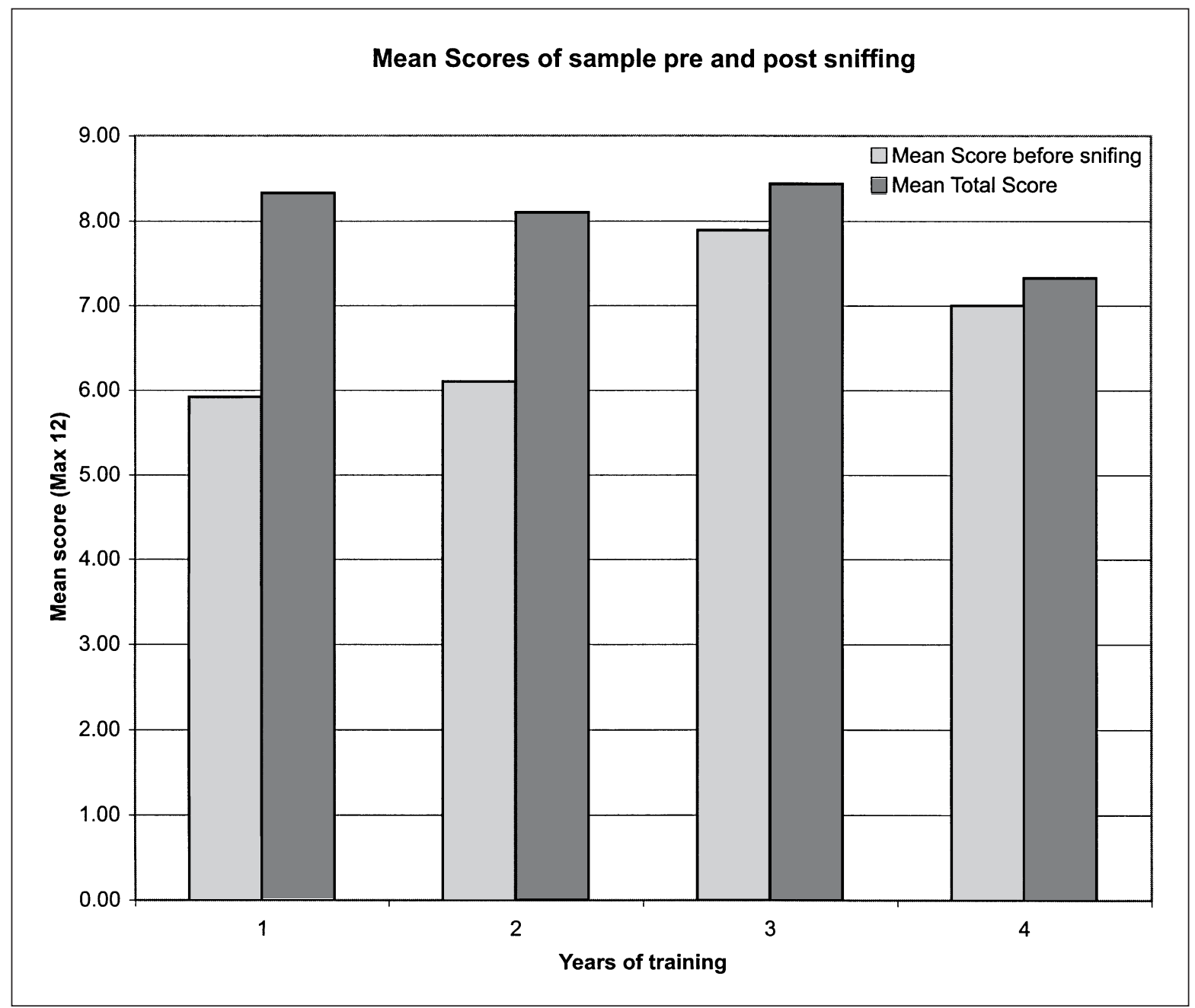

Figure 2: Scores divided by year of residency training of participants

\section{DISCUSSION}

We believe that a physician's sense of smell is an important tool for bedside diagnosis. The use of a Goldfrank sniffing bar improves the diagnostic accuracy of emergency medicine residents in an educational exercise using case histories to simulate acutely poisoned patients.

Improvement in accuracy was not uniformly distributed throughout the questions. The test subjects had a good rate of recognition (over $2 / 3$ of the subjects guessed correctly) with just the case history, before the use of the sniffing bar, in the questions involving cyanide, organophosphates, oil of wintergreen (methyl salicylate), and aromatic hydrocarbons (case numbers 1, 7,8 and 12). This may be because these poisonings are emphasized in emergency medicine curricula; the methyl salicylate question had a 97.6\% accurate response before the addition of the sniffing bar. With the exception of case number 7, the scores improved in these test cases after the addition of the sniffing bar anyway.
Responses to three of the cases did not change with addition of the sniffing bar (numbers 2, 7, and 11). Diagnostic accuracy did not change at all (9.5\% accuracy pre- and post-sniffing bar) in the test case involving macerated peanuts to simulate vacor (case number 4). This may be because vacor is a very unusual poisoning, it is not emphasized in the emergency medicine toxicology curriculum, and most emergency physicians are unfamiliar with its odor and properties.

Diagnostic accuracy did not change appreciably $(97.6 \%$ accuracy pre-sniffing bar and $95.2 \%$ after inclusion of the sniffing bar material) in the test case involving garlic oil to simulate organophosphate insecticide poisoning (number 7). We suspect that this is due to the emphasis placed on the recognition of organophosphates in our residency toxicology curriculum and resident familiarity with both the description of the clinical effects of organophosphate poisoning and the physical properties and odor of organophosphates.

Diagnostic accuracy did not change significantly (65\% before the sniffing bar and $67.5 \%$ after) in the case involving camphor 
mothballs to represent a pediatric ingestion of camphor. This was unexpected because camphor has a very characteristic smell and we thought that inclusion of the odor of camphor would improve diagnostic accuracy significantly even if the subject had no knowledge of the expected pathophysiology of camphor ingestion. Prior to this experiment, camphor poisoning was not emphasized in the toxicology curriculum of our residency program. Paradichlorobenzene and naphthalene mothballs are much easier to find in large department and convenience stores, but we purchased a large amount of pure $100 \%$ camphor mothballs in a small "dollar shop" in a predominantly Vietnamese neighborhood near our hospital. Despite a relatively high pre-sniffing bar diagnostic accuracy, the lack of recognition of the odor of camphor as evidenced by the low Likert confidence scores for this test case (CI 1.91-2.76) is a weakness in our toxicology teaching curriculum, in our opinion.

The only test case in which addition of the sniffing bar resulted in a significant decrease in diagnostic accuracy was the case involving household bleach to simulate the odor of chloramine gas. This, again, most likely reflects unfamiliarity of emergency medicine residents with the toxicity of chloramine gas, although this is a common poisoning and demonstrates a deficiency in our toxicology curriculum.

We found that the sniffing bar was a valuable instrument to demonstrate areas of weakness in our toxicology teaching curriculum. Perhaps the greatest value in the sniffing bar as a teaching tool may be for uncommon, rarely seen poisonings with characteristic odors. A topic for further study may be to use the sniffing bar tool as part of a teaching module on uncommon toxins, such as vacor, and then to test resident knowledge and accuracy in recognizing these unusual toxins using odors as an adjunct to the traditional case history. This conclusion concurs somewhat with the odor recognition experiment published by Rosenblatt et al., which concluded that "The recognition rates are higher for the more-common odors, and lower for the less-common odors. Teaching exposures to the less well-known odors are effective and can significantly improve the recognition rate of these substances."

\section{LIMITATIONS}

This was an observational, descriptive study and no sample size calculation was performed. A written case history can only approximate an actual patient encounter. Some toxins have easily recognizable odors that are difficult to recreate in a test tube. There may be substances and odors that better simulate the toxins being tested than the ones we used in our modified Goldfrank sniffing bar. Teaching modules with a sniffing bar may help reinforce the recognition and management of common or well known exposures, but they may not improve a test score during a simulated teaching module.

Just as some subjects have perfect hearing or vision and others need corrective lenses or hearing aids, some subjects may not have good olfactory appreciation. Testing subjects for smelling disorders prior to recruiting them for this experiment might have resulted in different outcomes. Similarly, subjects might have performed differently on the test if there had been an orientation or in-service on poisons and their odors prior to their participation in the experiment. Subjects were not taught to recognize the odor of the individual representative toxins; it was assumed that each subject is familiar enough with bleach, ammonia, and garlic to recognize the odors of each. All subjects bring their personal experiences to the test. This experiment may have different results if the subjects are trained in a location where they come into contact with industrial toxins such as cyanide, hydrogen sulfide, or glacial acetic acid frequently.

\section{CONCLUSION}

We found a modified Goldfrank sniffing bar to be a valuable adjunct for the teaching of toxicology to emergency medicine residents and for testing the comprehensiveness and timeliness of our toxicology curriculum. We recognize that we need to place more emphasis on common poisons and can probably deemphasize others. Further study into the use of this sniffing bar as part of a module on the recognition of uncommon yet characteristic toxins and their odors may expand the utility of the sniffing bar as a learning adjunct to the traditional toxicology teaching curriculum.

The authors have no potential financial conflicts of interest to report.

\section{REFERENCES}

1. Goldfrank L, Weisman R, Flomenbaum N. Teaching the recognition of odors. Ann Emerg Med 1982 Dec;11(12):684-686.

2. Rosenblatt Y, Phan P, Desandre P, Lobon L, Hsu C. Diagnostic odor recognition. Acad Emerg Med 2000 Oct; 7(10): 1168-1169.

3. In: Goldfrank L, Flomenbaum N, Lewin N et al., eds. Goldfrank's Toxicologic Emergencies, 8th ed. New York: McGraw-Hill, 2006.

4. In: Dart RC, Hurlbut K, Kuffner E, Yip L eds. The 5 Minute Toxicology Consult. Philadelphia: Lippincott Williams \& Wilkins, 2000. 